\title{
Acute interstitial nephritis after vaccination with BNT162b2
}

\author{
Sonja Rieckmann ${ }^{1} \cdot$ Felix S. Seibert $^{1} \cdot$ Maximilian Hogeweg $^{1} \cdot$ Sebastian Bertram $^{1} \cdot$ Adrian A. N. Doevelaar $^{1}$. \\ Kerstin Amann ${ }^{2} \cdot$ Nina Babel $^{1} \cdot$ Timm H. Westhoff ${ }^{1}$
}

Received: 7 December 2021 / Accepted: 6 February 2022 / Published online: 7 March 2022

(c) The Author(s) under exclusive licence to Italian Society of Nephrology 2022

Keywords COVID-19 · Vaccination · BNT162b2 · Kidney injury $\cdot$ Interstitial nephritis

\section{Case reports}

A 63-year-old male without any medical history was admitted to hospital because of malaise and anuria 3 weeks after his first vaccination with BNT162b2. The laboratory findings revealed acute kidney injury with an initial serum creatinine concentration of $19.0 \mathrm{mg} / \mathrm{dl}$, metabolic acidosis and hyperkalemia of $9 \mathrm{mmol} / \mathrm{l}$. Urinalysis showed nitrite negative leukocyturia, hematuria and proteinuria (albumin-tocreatinine ratio (ACR) $393 \mathrm{mg} / \mathrm{g}$, protein-to-creatinine ratio (PCR) $787 \mathrm{mg} / \mathrm{g}$ ). Serum creatinine concentration was in the normal range at his most recent laboratory examination. We performed a kidney biopsy, which revealed acute interstitial nephritis (AIN, Fig. 1A, B) with interstitial edema, lymphoplasmacellular interstitial infiltration with eosinophil granulocytes, and acute tubular necrosis. The patient denied having taken any medication. Anti-neutrophil antibodies (ANA), anti-neutrophil cytoplasmic antibodies (ANCA), IgG4, angiotensin-converting enzyme (ACE) and soluble IL2 receptor (sIL2R), immunofixation and hantavirus serology were unremarkable. RT-PCR from a nasopharyngeal swab specimen was negative for SARS-CoV-2. The patient denied any symptoms of COVID-19 in the past months. Thus, the SARS-CoV-2 vaccination was the only apparent trigger of the interstitial nephritis with acute kidney injury AKIN stage III. Initially, the patient required renal replacement therapy at an intensive care unit. Anti-inflammatory

Timm H. Westhoff

Timm.Westhoff@elisabethgruppe.de

1 Medical Department I, University Hospital Marien Hospital Herne, Ruhr-University Bochum, Hölkeskampring 40, 44625 Herne, Germany

2 Department of Nephropathology, Institute of Pathology, Friedrich-Alexander University (FAU) Erlangen-Nürnberg, Erlangen, Germany therapy was started with a daily dosage of $250 \mathrm{mg}$ prednisolone and was deescalated to a daily dosage of $80 \mathrm{mg}$ after three days. Diuresis $>500 \mathrm{ml}$ returned after 8 days, and hemodialysis was successfully discontinued after 2 weeks.

An 18-year-old male, also without any medical history, presented to his general practitioner for fatigue and cough, almost 6 weeks after his second BNT162b2 vaccination. Laboratory examinations showed an increase of serum creatinine concentration to $1.7 \mathrm{mg} / \mathrm{dl}$ and the young man was referred to our Department of Nephrology. Beyond a confirmation of an increased serum creatinine concentration, laboratory findings revealed hematuria, glycosuria and proteinuria (albumin-to-creatinine ratio (ACR) $393 \mathrm{mg} / \mathrm{g}$, protein-to-creatinine ratio (PCR) $787 \mathrm{mg} / \mathrm{g}$ ). Kidney biopsy was performed and provided a histology result resembling that of patient 1 , showing lymphoplasmacellular infiltration with eosinophil granulocytes and diffuse acute tubular necrosis consistent with AIN (Fig. 1C). In addition, immunohistochemistry disclosed mild mesangial IgA deposition without sclerosis, endocapillary proliferation or crescents (Fig. 1D). As was the case for patient 1, all laboratory parameters indicating a systemic disease underlying AIN, remained negative. Moreover, the patient was not taking any medication and denied symptoms of SARS-CoV-2 infection in the last few months. SARS-CoV-2 RNA PCR from a nasopharyngeal swab remained negative. Quantification analysis of antibodies to SARS-CoV-2 nucleocapsid protein were negative, thus making an asymptomatic SARS$\mathrm{CoV}-2$ infection in the past very unlikely. Hence, there were no other obvious triggers for AIN than the vaccine. After initiating steroid therapy with $50 \mathrm{mg}$ prednisolone per day, excretory renal function and tubular dysfunction recovered completely within 2 weeks.

Another patient, a 25-year-old female, also without prior illnesses, consulted her general practitioner 3 weeks after her third BNT162b2 vaccination, due to fatigue and general 

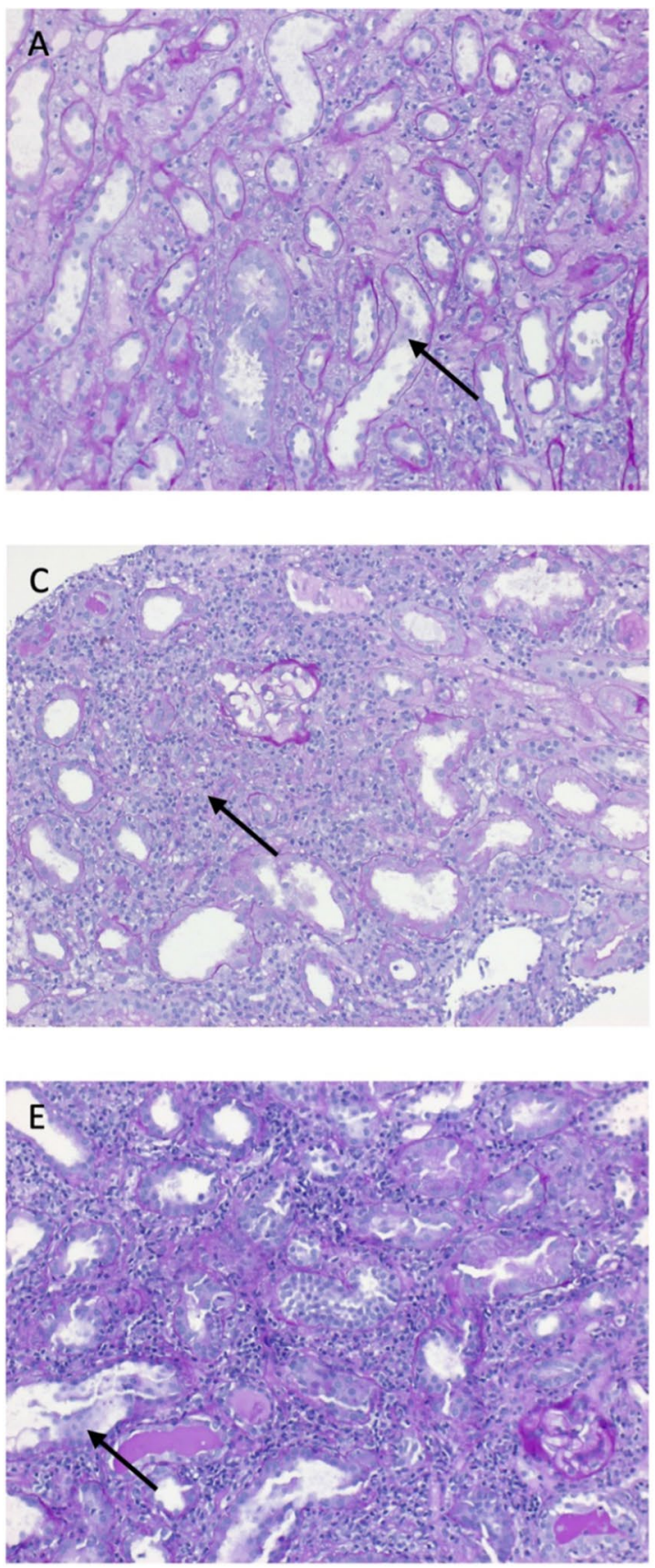

Fig. 1 Kidney biopsy of patient 1 in PAS staining showing $(\mathbf{A}, \times 20)$ acute tubular necrosis (arrow), interstitial edema and lymphoplasmacellular interstitial infiltration with few eosinophil granulocytes in $\mathrm{HE}$ staining $(\mathbf{B}, \times 40$, arrow), consistent with acute interstitial nephritis. Kidney biopsy of patient 2 in PAS staining showing $(\mathbf{C}, \times 20)$ dense lymphoplasmacellular infiltration (arrow) with eosinophil granulocytes and diffuse acute tubular necrosis consistent with acute inter-

malaise. Laboratory findings showed acute kidney injury with initial serum creatinine concentration of $11.7 \mathrm{mg} /$ dl. Therefore, the young patient was admitted to our Department of Nephrology.
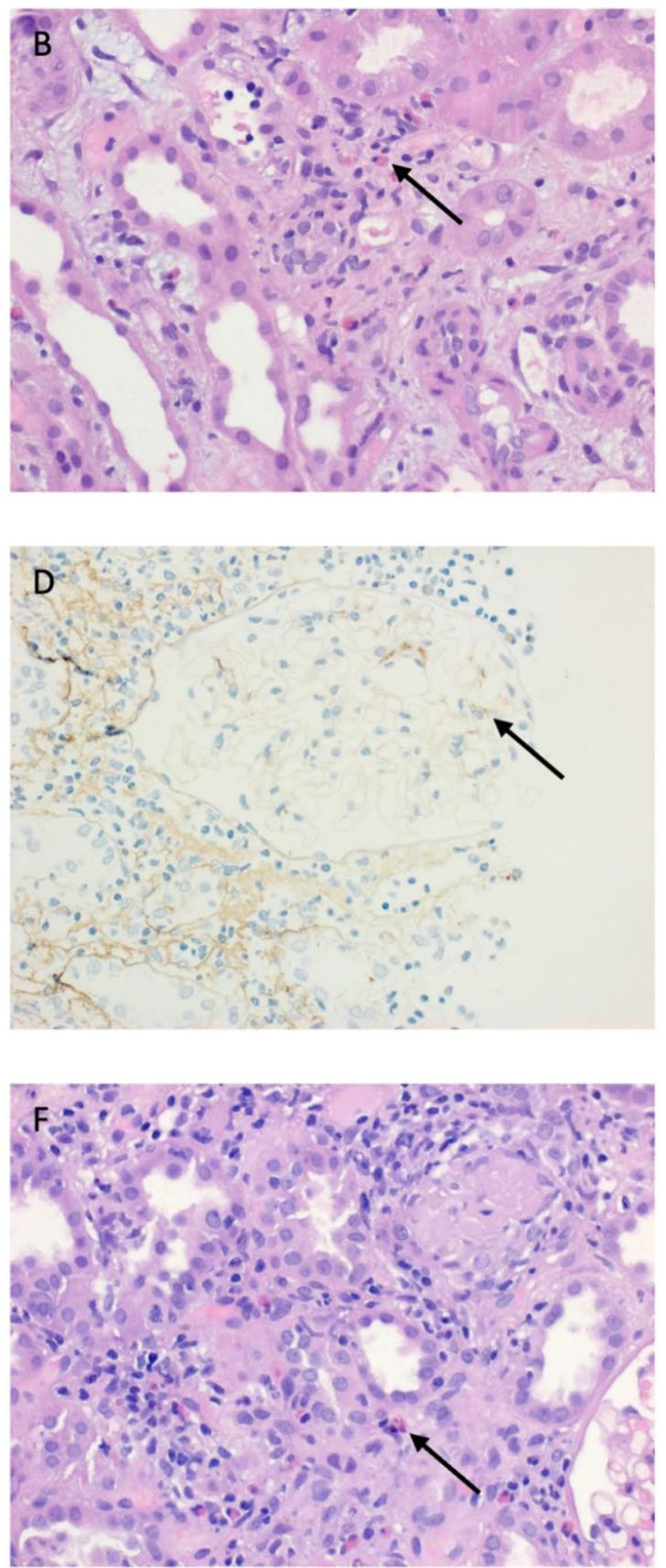

stitial nephritis as well as a normal glomerulus. Immunohistochemistry $(\mathbf{D}, \times 40)$ revealed mild granular mesangial IgA deposition (arrow) in an otherwise normal glomerulus. Kidney biopsy of patient 3 in PAS staining showing $(\mathbf{E}, \times 20)$ severe, locally destructive interstitial nephritis with prominent diffuse acute tubular necrosis (arrow) and slight eosinophilia in HE staining $(\mathbf{F}, \times 40$, arrow $)$

Urinalysis revealed hematuria, glycosuria and proteinuria (albumin-to-creatinine ratio (ACR) $58 \mathrm{mg} / \mathrm{g}$, protein-tocreatinine ratio (PCR) $235 \mathrm{mg} / \mathrm{g}$ ). Histopathologic examination of kidney biopsy material showed severe, locally destructive interstitial nephritis with prominent diffuse acute 
tubular necrosis (Fig. 1E) and slight eosinophilia (Fig. 1F). Similarly to patients 1 and 2, all laboratory values related to a systemic disease with etiological connection to AIN yielded negative results. Drug history was unremarkable, symptoms of COVID-19 were denied. A nasopharyngeal swab for SARS-CoV-2 RT-PCR remained negative, as did supplemental analysis of SARS-CoV-2 nucleocapsid antibodies, thus we concluded that BNT162b2 vaccination was the only apparent trigger for AIN with acute kidney injury AKIN stage III. We initiated anti-inflammatory therapy with $250 \mathrm{mg}$ prednisolone per day, deescalated to a daily dosage of $80 \mathrm{mg}$ after three days with rapid response to treatment and progressive recovery of excretory renal function within days.

\section{Lessons for the clinical nephrologist}

Current mRNA-based vaccination strategies for SARSCoV-2 have proven high efficacy and a very low incidence of potentially serious side effects. Immune-mediated specific side effects have primarily been described for the heart. Thus, the incidence of myocarditis among young male recipients is increased after receiving the BNT162b2 vaccine, particularly after the second dose [1]. Vaccines might potentially also trigger new onset or exacerbation of inflammatory tubulointerstitial or glomerular kidney diseases. In contrast to myocarditis, however, data on renal side effects after administration of SARS-CoV-2 vaccines are scarce.

Here, we report three cases of biopsy proven AIN in association with SARS-CoV-2 vaccination with BNT162b2. History and laboratory findings did not reveal any alternative etiologic factors. There were neither typical causes of acute interstitial nephritis like drugs or infections, nor a systemic entity like IgG4 disease or sarcoidosis or a former SARS$\mathrm{CoV}-2$ infection. Histology findings were similar in all three subjects and shared infiltration of eosinophils, suggestive of, e. g., drug-induced AIN. In the absence of any alternative drug intake, this might support a potential etiological role of the vaccine. All acute kidney injuries were rapidly responsive to steroids and recovered completely within 2 weeks after prednisolone therapy.

AIN is one of the major causes of acute kidney injury, and it is frequently triggered by drug therapy $(80 \%)$ or infectious agents. It is driven by an immune-mediated inflammatory process, histopathologically characterized by the presence of inflammatory infiltrates, mostly composed of T- and B-lymphocytes, macrophages, eosinophils and natural killer cells, resulting in local edema, disruption of the tubular basement membrane and destruction of the interstitial architecture. Immunologic reactions are induced by the expression of endogenous or exogenous antigens processed by tubular cells [2].
The high number of millions of vaccinated individuals during the COVID-19 pandemic offers the opportunity to identify even very rare side effects that did not become evident during the phase III trials. In recent literature, there are only isolated case reports on associations between SARS$\mathrm{CoV}-2$ immunization and kidney disease. There is a case series on glomerular diseases becoming clinically manifest after vaccination [3]. The authors describe IgA nephropathy, minimal change disease, membranous nephropathy, crescentic glomerulonephritis, and collapsing glomerulonephritis. With regard to collapsing glomerulopathy in the context of COVID-19, those patients with collapsing glomerulopathy were of African American descent and had APOL1 genomic risk alleles. The new onset or exacerbation of glomerular diseases has been described for both mRNA and adenoviral vaccines against COVID-19 [4, 5]. Regarding all these glomerular diseases, it may be assumed that the vaccination constituted an inflammatory stimulus, which triggered the clinical onset of a glomerular disease in individuals with an underlying predisposition. Vaccination as a trigger of autoimmune disease is a well characterized phenomenon in a broad variety of entities including encephalitis disseminata and systemic lupus erythematosus.

In contrast to the association of SARS-CoV-2 vaccination with flairs of glomerular disease, the association with interstitial renal disease has not been identified as a clinical problem so far. To the best of our knowledge, there are just three reports, the first of which was published in June 2021 [6], each describing just one case of interstitial nephritis in temporal association with administration of BNT162b2. These reports, however, do not describe a complete etiological work-up of alternative etiologies of AIN.

The mechanisms underlying this very rare association of glomerular or interstitial renal disease with vaccination to SARS-CoV-2 remain elusive. In this context, however, molecular mimicry, which refers to a similarity between structures of the vaccine and human proteins with consecutive immune cross-reactivity, constitutes a candidate mechanism [7]. If so, it would be of interest to establish whether AIN occurs in COVID-19 as well. Indeed, AIN has been described as a histological finding in COVID-19 patients [8].

While the principle of eliciting immune response differs- on the one hand between the different vaccines (virus vector or lipid nanoparticles) and on the other between infection and vaccine-the receptor-binding domain of S1 subunit of the SARS-CoV-2 spike protein, which facilitates intracellular virus entry, is the immunodominant target of neutralizing antibodies in both infected patients and vaccinated people [9]. Further studies are needed to identify the responsible antigen.

The present case reports describe AIN as a rare, but potentially serious side effect of mRNA vaccination to SARS-CoV-2. Whereas the course of AIN was mild in 
patient 2, it was associated with severe acute kidney failure in patients 1 and 3. Physicians should be aware of this rare, but potentially serious complication and its excellent responsiveness to steroid treatment.

Funding No funding has been received for this study.

\section{Declarations}

Conflict of interest The authors have declared no conflicts of interest.

Ethical approval The article does not contain any studies with human participants performed by any of the authors.

Informed consent The patients provided informed consent.

\section{References}

1. Mevorach D, Anis E, Cedar N et al (2021) Myocarditis after BNT162b2 mRNA vaccine against Covid-19 in Israel. N Engl J Med 385:2140-2149. https://doi.org/10.1056/NEJMoa2109730

2. Praga M, Gonzalez E (2010) Acute interstitial nephritis. Kidney Int 77:956-961. https://doi.org/10.1038/ki.2010.89

3. Tiffany N, Caza CAC, Messias N, Hannoudi A et al (2021) Glomerular disease in temporal association to SARS-CoV-2 vaccination-a series of 29 cases. Kidney360 2:1770-1780. https://doi.org/10.34067/KID.0005372021

4. Leclerc S, Royal V, Lamarche C et al (2021) Minimal change disease with severe acute kidney injury following the OxfordAstraZeneca COVID-19 vaccine: a case report. Am J Kidney Dis 78:607-610. https://doi.org/10.1053/j.ajkd.2021.06.008

5. Abramson M, Mon-Wei YuS, Campbell KN et al (2021) IgA nephropathy after SARS-CoV-2 vaccination. Kidney Med 3:860 863. https://doi.org/10.1016/j.xkme.2021.05.002

6. de la Flor J, Linares T, Alonso-Riano M et al (2021) A case of acute interstitial nephritis following the Pfizer-BioNTech COVID19 vaccine. Nefrologia (Engl Ed). https://doi.org/10.1016/j.nefro. 2021.05.004

7. Segal Y, Shoenfeld Y (2018) Vaccine-induced autoimmunity: the role of molecular mimicry and immune crossreaction. Cell Mol Immunol 15:586-594. https://doi.org/10.1038/cmi.2017.151

8. Ng JH, Bijol V, Sparks MA et al (2020) Pathophysiology and pathology of acute kidney injury in patients with COVID-19. Adv Chronic Kidney Dis 27:365-376. https://doi.org/10.1053/j.ackd. 2020.09.003

9. Chen EC, Gilchuk P, Zost SJ et al (2021) Convergent antibody responses to the SARS-CoV-2 spike protein in convalescent and vaccinated individuals. Cell Rep 36:109604. https://doi.org/10. 1016/j.celrep.2021.109604

Publisher's Note Springer Nature remains neutral with regard to jurisdictional claims in published maps and institutional affiliations. 